\title{
Optimization of a 12.5 GHz Microstrip Antenna Array Using Taguchi's Method
}

\author{
M. Spasos, ${ }^{1,2}$ R. Nilavalan, ${ }^{2}$ K. Tsiakmakis, ${ }^{1}$ N. Charalampidis, ${ }^{1}$ and S. W. Cheung ${ }^{3}$ \\ ${ }^{1}$ Department of Electronics, Alexander Technological Educational Institute of Thessaloniki, P.O. Box 141, 57400 Sindos, Greece \\ ${ }^{2}$ Department of Electronic and Computer Engineering, Brunel University, London, Uxbridge, Middlesex UB8 3PH, UK \\ ${ }^{3}$ Department of Electrical and Electronic Engineering, The University of Hong Kong, Room 601, Chow Yei Ching Building, \\ Pokfulam Road, Hong Kong \\ Correspondence should be addressed to M. Spasos, spasos@el.teithe.gr
}

Received 20 April 2011; Revised 17 July 2011; Accepted 17 July 2011

Academic Editor: Hon Tat Hui

Copyright ( $) 2011$ M. Spasos et al. This is an open access article distributed under the Creative Commons Attribution License, which permits unrestricted use, distribution, and reproduction in any medium, provided the original work is properly cited.

This paper presents Taguchi's optimization method implemented in the design of a single feed (without any matching network) $5 \times 5$ microstrip antenna array operating around $12.5 \mathrm{GHz}$. The proposed optimization method is statistical and is widely used for quality assurance in many fields such as mechanical and chemical production, consumer electronics, services; however it has been underused in the field of electromagnetics. It allows optimization of multiparameter, multitarget complex designs in a very short time in conjunction with advanced simulation tools. The proposed antenna has been fully evaluated under Taghuchi's and PSO's optimization methods, and the experimental results show total Gain of $15 \mathrm{~dB}$, and good matching with $S 11$ better than $-20 \mathrm{~dB}$, in the frequency range 12.3 to $12.8 \mathrm{GHz}$.

\section{Introduction}

The demands of modern telecommunications increase hence, more complex antenna array configurations are required. Optimization and synthesis of microwave antenna arrays and other complex electromagnetic-based structures typically deal with multiobjective functions that are highly nonlinear, discontinuous, and have a large number of optimization parameters. In addition, due to strong mutual coupling and other propagation effects, they require a simultaneous optimization of the design parameters.

Recently, many multiagent stochastic optimization techniques that incorporate random variation and selection, such as evolutional programming (EPs) $[1,2]$, genetic algorithms (GA) $[3,4]$, particle swarm optimization (PSO) [5, 6], artificial neural network (ANN) [7, 8], and gradient-based techniques $[9,10]$, have been implemented via computer codes.

The above optimization methods can be divided into two categories: global and local techniques. Global techniques such as EP, GA, PSO, and ANN are capable of handling multidimensional, discontinuous, and nondifferentiable objective functions with many potential local maxima while they are largely independent of initial conditions. However, a main drawback is that the convergence rate is slow [10].

In contrast, the main advantage of local techniques such as the gradient-based algorithms is that the solution converges rapidly. However, local techniques work well only for a small number of continuous parameters highly depending on the starting point or the initial guessing while they reacting are relatively poorly in case of discontinuities in solution spaces $[10]$.

In order to bridge the weak points of these two techniques a new statistical optimization method is necessary. A way of achieving target optimization and reduction in variation around the target is to apply the Design of Experiment (DOE) technique [11]. DOE is a powerful statistical technique for improving product or process design as well as for solving production problems. A standardized version of the DOE has been introduced by Taguchi, an easy to learn and apply technique for design optimization and production problem investigation $[12,13]$. Taguchi's optimization technique can handle multidimensional, discontinuous, and 
nondifferentiable objective functions with many potential local maxima whilst converges rapidly to the optimum result but within a well-defined area [14].

The goal of Taguchi's method is the prediction of the parameters that cause variability (noise) and the optimum value of each parameter (control), in order to achieve robustness of the device under test. The Taguchi approach has the great advantage that only a small fraction of all possible parameter-level combinations is tested, based on the theory of highly fractional orthogonal designs (orthogonal arrays).

Previously published work in the field of antenna array using Taguchi's technique had as optimization goal either pattern or input matching criteria. Work on radiation patterns includes optimization of the spiral antenna gain [15] as well as optimizing linear arrays for nulls at specific directions, suitable pattern shapes, and suppressed side lobes [16]. Work on input matching includes two-layer wideband patch antenna [17], ultrabroadband zigzag logperiodic antenna [18], and a CPW slot antenna [11]. This paper presents, for the first time, the optimization of a simple microstrip planar $5 \times 5$ antenna array configuration with interconnected elements in order to achieve good matching $(S 11<-10 \mathrm{~dB})$ without using of any matching networks and high gain, suitable for the $12.5 \mathrm{GHz}$ broadcasting satellite service (BSS) frequency bands.

The exact dimensions of the array are precisely calculated by applying a Taguchi's optimization algorithm in conjunction with an electromagnetic solver FEKO [19]. The objective of Taguchi's algorithm is the minimization of a particular mathematical "fitness function," which is suitably determined according to the criteria mentioned above.

Furthermore, a statistical analysis of variance (ANOVA) of the optimized dimensions of the antenna is applied to the final iteration. The goal of this analysis is the investigation of the significance of each parameter within their final limits in order to fabricate a robust device.

\section{Taguchi Optimization Method}

The optimization procedure begins after some initial consideration as regards the initial conditions setting as well as the selection of a proper $\mathrm{OA}$ and an appropriate expression of the fitness function (FF). The selection of an OA depends on the number of input parameters and the number of levels for each parameter. The FF is a particular mathematical function and is developed according to the nature of the problem and the optimization goals.

After a simple analysis, the simulation results serve as objective functions for optimization and data analysis, and an optimum combination of the parameter values can be obtained. The log functions of the outputs, named by Taguchi as Signal-to-Noise ratios $(S / N)$, are used for the prediction of the optimum result. It can be demonstrated via statistics that although the number of experiments are dramatically reduced, the optimum result obtained through the orthogonal array usage is very close to that obtained by making use of the full factorial approach.

When the Taguchi method is implemented at the design level and the efforts are focused on the optimization of the control values, the experiments can be replaced with simulations.

In order to achieve as high convergence with the goal as possible, successive implementations of the method have to be applied. Under this procedure, the optimum results of the last iteration serve as central values for the next, reducing each time with a predefined factor the level difference of each parameter. The procedure terminates when the level difference becomes negligible, and maximum available accuracy has been reached.

The procedural steps in detail are shown below.

(1) Consideration of the problem that must be solved.

(2) Extraction of the FF and definition of the optimum goal (minimum, nominal, or maximum).

(3) Definition of the main parameters and their estimated (center) values.

(4) Definition of the levels limit for each parameter within $\pm 10 \%$ of the center values. In order to describe the nonlinear effect so as to gradually minimize each iteration level's difference, an odd number of levels must be used for each input parameter.

(5) Definition of the maximum resolution of the parameters.

(6) Design of experiment (DOE) using Taguchi's suggested orthogonal arrays $\mathrm{OAn}(\mathrm{mk})$ in order to minimize the effect of any erroneous assumptions that have been made due to effects considered negligible, which consist of

(i) $n$ rows (number of experiments),

(ii) $k$ columns (number of parameters), and

(iii) $m$ levels (on which each parameter will vary).

(7) Simulation using FEKO according to the selected OA.

(8) Evaluation of the compliance of the FF for each combination of the levels of parameters based on the simulation results.

(9) Computation of the mean value of the fitness functions of the experiment

$$
\bar{Y}=\frac{1}{n} \sum_{i=1}^{i=n} Y_{i}
$$

(10) Computation of the mean value for each level of each parameter

$$
\bar{Y}_{m_{i}}=\frac{m}{n} \sum_{i=1}^{i=n / m} Y_{m_{i}} .
$$

(Example: for the parameter $A$ when level is 1, add the values of all corresponding FF and compute their mean value).

(11) Consideration of the optimum level for each parameter depending on the $\bar{Y}_{m_{i}}$ and the nature of the goal (minimum, nominal, or maximum). 
(12) Prediction of the optimum value of the experiment's FF, based on the $20 \cdot \log 10$ values of $\bar{Y}$ and the $\bar{Y}_{m_{i}}$. (The conversion is essential in order to avoid negative values especially at the beginning, when the differences between $\bar{Y}$ and $\bar{Y}_{m_{i}}$ are high)

$$
\begin{aligned}
Y_{O(\log )}= & \bar{Y}_{(\log )}-\left(\bar{Y}_{(\log )}-\bar{Y}_{\left.1 m_{\mathrm{opt}(\log )}\right)}\right) \\
& -\cdots\left(\bar{Y}_{(\log )}-\bar{Y}_{\left.k m_{\mathrm{opt}(\log )}\right)}\right) .
\end{aligned}
$$

The predicted value might not be the optimum because the $\mathrm{OA}$ is a fractional factorial design, but, nevertheless, it shows the direction of the optimization. During the next iterations, as the gap between the mean and optimum predicted value becomes smaller, the possibility that the optimum predicted value to be the real optimum value rises significantly.

(13) Definition of the reducing percentage (RP) of the initial difference between the levels of the parameters. The RP depends on the nature of the problem and can be high for simple cases with only one optimum condition or low for more complex situations.

(14) Creation of new level differences (LDs) by multiplying the RP with the initial level of the parameters

$$
\mathrm{LD}_{i}=\mathrm{L}_{\text {init }} \cdot(1-\mathrm{RP}) .
$$

(15) Creation of new levels for the next iteration by adding the estimated optimum levels of the parameters of the 1 th iteration with the $\mathrm{LD}_{i}$.

(16) The procedure stops when the $\mathrm{LD}_{i}$ reaches the limits of the allowed resolution of the parameters.

(17) Decision, on which parameters are significant based on their final levels, using statistical analyses of variance (ANOVA).

(18) Construction of the antenna taking special care of the significant parameters as they will be responsible for any variability in the antenna characteristics.

\section{Design of the Antenna}

A $5 \times 5$ microstrip planar array is considered for the study. The array is tuned to work for the $12.5 \mathrm{GHz}$ Broadcasting Satellite Service (BSS) frequency bands. This antenna array consists of equally spaced rectangular patches joined together with microstrip lines and is fed through a simple microstrip line without any matching network, as shown in Figure 1. In this paper, Taguchi's method will be used to optimize the geometrical dimensions of the antenna so as to simultaneously achieve good matching, low side lobe level, and high gain in the operating frequency range.

The substrate chosen for the antenna implementation is the microwave laminate RT/duroid 5880 from Rogers with er $=2.2, \tan \delta=0.0009$, and thickness of $1.575 \mathrm{~mm}$.

The objective of Taguchi's algorithm is the minimization of the FF. The FF is suitably determined according to the

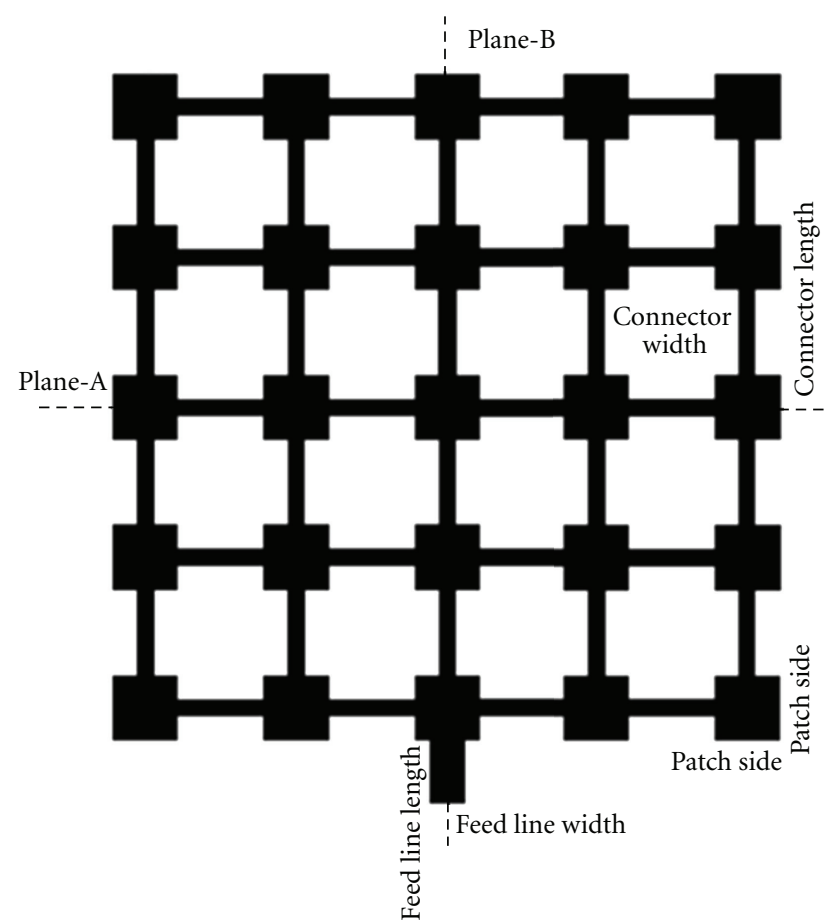

Figure 1: $5 \times 5$ microstrip antenna array.

above three conditions. The goals that have to be achieved are

(i) good input matching $S 11<-10 \mathrm{~dB}$,

(ii) high gain $>10 \mathrm{~dB}$ in the pattern cut $\varphi j(0 \leq \varphi j \leq 2 \pi)$, $\theta j\left(-5^{\circ} \leq \theta j \leq 5^{\circ}\right)$,

(iii) low side lobe in the pattern cut $\varphi j(0 \leq \varphi j \leq 2 \pi)$, $\theta j\left(15^{\circ} \leq \theta j \leq 90^{\circ}\right)$, and $\theta j\left(-90^{\circ} \leq \theta j \leq-15^{\circ}\right)$, SLL (difference between main lobe and secondary lobe) $>10 \mathrm{~dB}$.

Thus, a weighted FF has been chosen with the form

$$
\begin{aligned}
\mathrm{ff}= & 1000 \cdot\left(15-\left|S 11_{(\mathrm{dB})}\right|\right) \\
& +1000 \cdot\left(15-\operatorname{Gain}_{(\mathrm{dB})}\right)+\left(250 \cdot \mathrm{SLL}_{(\mathrm{dB})}\right),
\end{aligned}
$$

where the weight factors $(1000,250)$ indicate the significance of the targets, and factor 15 indicates the optimum result (goal $+50 \%)$ for input matching and gain. There is also a dead zone in the pattern cut $\varphi j(0 \leq \varphi j \leq 2 \pi), \theta j\left(6^{\circ} \leq \theta j \leq\right.$ $\left.14^{\circ}\right)$, and $\theta j\left(-14^{\circ} \leq \theta j \leq-6^{\circ}\right)$ where the results are ignored as they are irrelevant to gain or SLL results.

It has to be mentioned that the computation takes into account the case of the first two factors being negative, that is, $\left|S 11_{(\mathrm{dB})}\right|>15$ and $\mathrm{Gain}_{(\mathrm{dB})}>15$ and keeps them to zero otherwise; the FF could be driven to false results, discriminating towards one or the other characteristic.

In the design of an array antenna, the most important design parameters are usually the number of elements, spacing between the elements, excitation (amplitude and phase), half-power beamwidth, directivity, and side lobe level 
TABLE 1: The initial levels for the optimization procedure.

\begin{tabular}{lccc}
\hline Parameters & 1st Level & 2nd Level & 3rd Level \\
\hline (A) Patch side & 9.6 & 12 & 14.4 \\
(B) Connector width & 2.4 & 3 & 3.6 \\
(C) Connector length & 14.4 & 18 & 21.6 \\
(D) Line Feed width & 4.8 & 6 & 7.2 \\
(E) Line Feed length & 9.6 & 12 & 14.4 \\
\hline
\end{tabular}

[20, 21]. Hence, the initial center dimensions for each parameter can be defined as shown below.

The free air wavelength at $12.5 \mathrm{GHz}$ is $\lambda_{0}=24 \mathrm{~mm}$.

(A) Patch side $=\lambda_{0} / 2=12 \mathrm{~mm}$

(B) Connector length $=3 \lambda_{0} / 4=18 \mathrm{~mm}$

(C) Connector width $=\lambda_{0} / 8=3 \mathrm{~mm}$

(D) Feeding line length $=\lambda_{0} / 2=12 \mathrm{~mm}$

(E) Feeding line width $=\lambda_{0} / 4=6 \mathrm{~mm}$

Considering $a \pm 20 \%$ deviation from the center values, initial levels can be created, as shown in Table 1. For a realistic approach in the antenna design, the resolution for all dimensions is set to $1 \mu \mathrm{m}$. For an OA with 5 parameters of 3 levels for each parameter, a configuration with at least $n_{\text {rows }}=1+\left(k \cdot \mathrm{DOF}_{m}\right)=1+(5 \cdot 2)=11$ rows is needed. Taguchi suggests two solutions

(i) the $\mathrm{OA}_{18}(37,2)$ that can handle up to 7 parameters with 3 levels each and one with 2 levels in an array of 18 rows.

(ii) the $\mathrm{OA}_{27}(313)$ that can handle up to 13 parameters with 3 levels each in an array of 27 rows.

Since achieving the desired gain and obtaining good matching is a complex task, it was decided to employ a larger OA to increase the possible combinations. Thus, the second solution appears more promising (Table 2) as it has $50 \%$ more rows for each $\mathrm{OA}$ and offers higher confidence level at the expense of some extracomputation time $(11.5 \mathrm{~h}$ instead of $7.5 \mathrm{~h}$ for this case study).

According to the complexity of the design, a reducing percentage of $\mathrm{RP}=0.1$ has been assigned. The procedure terminates when the LD is below $1 \mu \mathrm{m}$ (minimum resolution) after twenty-five optimization cycles (27 each), finally reaching 675 iterations.

FEKO is a software suite built for the analysis of a wide range of electromagnetic problems. Applications include EMC analysis, antenna design, microstrip antennas and circuits, dielectric media, and scattering analysis.

The core of the software package is based on the method of moments (MoMs). The MoM is a full wave solution of Maxwell's integral equations in the frequency domain. The active area of the microstrip array antenna design of this case study consists of 25 metallic, equal in size, squared patches separated by equal connector lines, and a single microstrip feeding line as shown in Figure 1. The calculation of the antenna elements as well as their simulation has been carried

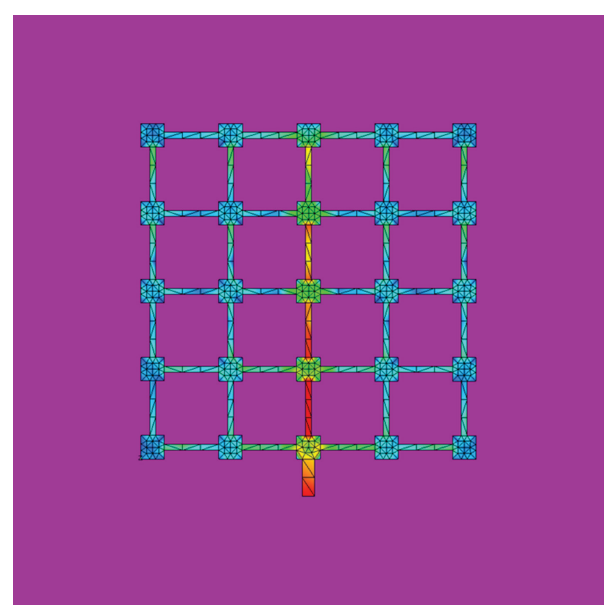

Figure 2: FEKO-meshed design of the array antenna with current distribution.

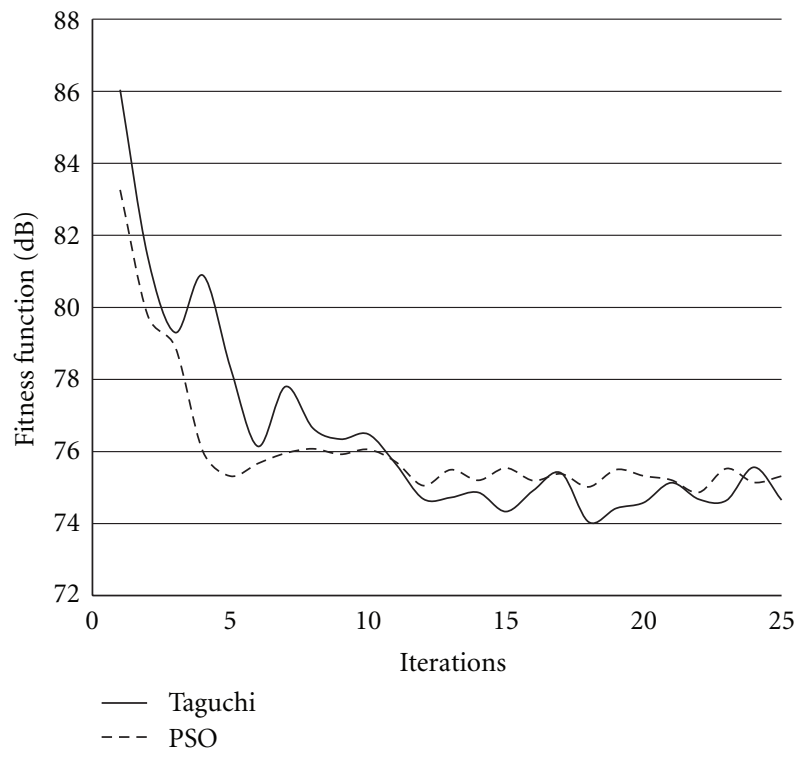

FIGURE 3: Taguchi and PSO optimization procedure graphs.

out in EDITFEKO module, which is capable of simplifying the process of generating and editing * ${ }^{*}$.pre (PREFEKO input) files as this is a standard text editor that can use ASCII text files with customized functionality. For such a complicated iterative design, the editor EDITFEKO is chosen to simplify the process of generating and editing * ${ }^{*}$.pre (PREFEKO input) files as this is a standard text editor that can use ASCII text files with customized functionality. After meshing the active area of the antenna has been separated into 944 triangles, as illustrated in Figure 2.

To model the dielectric area under the metallic patches, FEKO uses Green's function formulation which implements $2 \mathrm{D}$ infinite planes with finite thickness $(h=1.575 \mathrm{~mm}$, er $=$ $2.2, \tan \delta=0.0009$ ) to handle the dielectric layer. Under the dielectric layer, real ground layer is modeled using reflection coefficient approximation to complete the microstrip antenna design. 
TABLE 2: The $\mathrm{OA}_{27} 5^{3}$ as configured with the parameters levels and the responses of the final FF.

\begin{tabular}{|c|c|c|c|c|c|c|}
\hline$n$ rows & A Patch side & B Con. length & C Con. width & D Feed. line length & E Feed. line width & FF Resp. FF (dB) \\
\hline 1 & 1 & 1 & 1 & 1 & 1 & 76.16 \\
\hline 2 & 2 & 2 & 2 & 2 & 2 & 75.14 \\
\hline 3 & 3 & 3 & 3 & 3 & 3 & 75.51 \\
\hline 4 & 2 & 2 & 3 & 3 & 3 & 75.35 \\
\hline 5 & 3 & 3 & 1 & 1 & 1 & 75.20 \\
\hline 6 & 1 & 1 & 2 & 2 & 2 & 75.98 \\
\hline 7 & 3 & 3 & 2 & 2 & 2 & 75.26 \\
\hline 8 & 1 & 1 & 3 & 3 & 3 & 76.40 \\
\hline 9 & 2 & 2 & 1 & 1 & 1 & 75.04 \\
\hline 10 & 2 & 3 & 1 & 2 & 3 & 74.92 \\
\hline 11 & 3 & 1 & 2 & 3 & 1 & 76.02 \\
\hline 12 & 1 & 2 & 3 & 1 & 2 & 75.35 \\
\hline 13 & 3 & 1 & 3 & 1 & 2 & 76.09 \\
\hline 14 & 1 & 2 & 1 & 2 & 3 & 75.05 \\
\hline 15 & 2 & 3 & 2 & 3 & 1 & 74.93 \\
\hline 16 & 1 & 2 & 2 & 3 & 1 & 75.14 \\
\hline 17 & 2 & 3 & 3 & 1 & 2 & 75.84 \\
\hline 18 & 3 & 1 & 1 & 2 & 3 & 76.06 \\
\hline 19 & 3 & 2 & 1 & 3 & 2 & 75.14 \\
\hline 20 & 1 & 3 & 2 & 1 & 3 & 74.92 \\
\hline 21 & 2 & 1 & 3 & 2 & 1 & 76.40 \\
\hline 22 & 1 & 3 & 3 & 2 & 1 & 75.83 \\
\hline 23 & 2 & 1 & 1 & 3 & 2 & 76.16 \\
\hline 24 & 3 & 2 & 2 & 1 & 3 & 75.23 \\
\hline 25 & 2 & 1 & 2 & 1 & 3 & 75.99 \\
\hline 25 & 3 & 2 & 3 & 2 & 1 & 75.08 \\
\hline 27 & 1 & 3 & 1 & 3 & 2 & 74.92 \\
\hline
\end{tabular}

Finally POSTFEKO module is used to visually confirm the correctness of the model before starting a potentially time demanding FEKO simulation. It is also used to display the results, of the FEKO simulation, either in the form of position in a $3 \mathrm{D}$ window or in arbitrary $2 \mathrm{D}$ graphs.

Taking into account the above considerations in combination with FEKO simulation results the Taguchi's optimization algorithm for the $5 \times 5$ antenna array of Figure 1 is implemented in $\mathrm{C}++$. The use of $\mathrm{C}++$ code offers the ability to alter the antenna's parameters since all the parameters can be entered in a text format and get processed by the program.

\section{Results}

For comparison reasons, the parameters of the antenna will also be optimized using the well-known Particle Swarm Optimization (PSO) method. The particles and the FF of the PSO are set to be the same as those of Taguchi's method. Therefore, the optimization performance of both optimizers could be compared to each other according to the total number of iterations required towards the design goal.

For a fare comparison, 675 iterations are also conducted by PSO choosing the best FF every after 27 iterations to compare it with that of Taguchi's method.
TABLE 3: The optimized values of the parameters.

\begin{tabular}{lc}
\hline (A) Patch side & $10.823 \mathrm{~mm}$ \\
(B) Connector width & $2.498 \mathrm{~mm}$ \\
(C) Connector length & $16.569 \mathrm{~mm}$ \\
(D) Line feed length & $11.196 \mathrm{~mm}$ \\
(E) Line feed width & $6.875 \mathrm{~mm}$ \\
\hline
\end{tabular}

The optimization procedure graph, shown in Figure 3, presents the curves of the best FF values for every optimization cycle, as they converged under Taguchi and PSO processes. The results show that Taguchi's method excels PSO (some $1 \mathrm{~dB}$ less as both algorithms are configured as minimizers), when a well-defined narrow searching area is under consideration.

The results for optimum dimensions extracted after 25 cycles of iterations (less than 4 hours of processing time) for the $12.5 \mathrm{GHz}$ single feed planar antenna array are illustrated in Table 3.

In order to fabricate a good performance and robust design taking into account the fabrication tolerances, the selected $\mathrm{OA}_{27}$ (35) with the final results are examined under 
TABLE 4: The analysis of variance (ANOVA) table.

(a) General linear model: $12.5 \mathrm{GHz}$ antenna array versus A; B; C; D; E

\begin{tabular}{llcc}
\hline Factor & Type & Levels & Values \\
\hline A & Fixed & 3 & $1 ; 2 ; 3$ \\
B & Fixed & 3 & $1 ; 2 ; 3$ \\
C & Fixed & 3 & $1 ; 2 ; 3$ \\
D & Fixed & 3 & $1 ; 2 ; 3$ \\
E & Fixed & 3 & $1 ; 2 ; 3$ \\
\hline
\end{tabular}

(b) Analysis of variance for $12.5 \mathrm{GHz}$ antenna array, using adjusted SS for tests

\begin{tabular}{|c|c|c|c|c|c|c|}
\hline Source & $\begin{array}{c}\text { DF } \\
\text { degrees of freedom }\end{array}$ & $\begin{array}{c}\text { Seq SS } \\
\text { Seq/al sums } \\
\text { squares }\end{array}$ & $\begin{array}{c}\text { Adj SS } \\
\text { adjusted sums squares }\end{array}$ & $\begin{array}{c}\text { Adj MS } \\
\text { adjusted means } \\
\text { squares }\end{array}$ & $\begin{array}{c}\mathrm{F} \\
F \text {-statist Adj MS/P }\end{array}$ & $\begin{array}{c}\mathrm{P} \\
P \text {-value }\end{array}$ \\
\hline A & 2 & 1340 & 1340 & 670 & 0.05 & 0.950 \\
\hline B & 2 & 1592002 & 1592002 & 796001 & 61.68 & 0.000 \\
\hline $\mathrm{C}$ & 2 & 230663 & 230663 & 15332 & 8.94 & 0.002 \\
\hline D & 2 & 776 & 776 & 388 & 0.03 & 0.970 \\
\hline $\mathrm{E}$ & 2 & 3383 & 3383 & 1691 & 0.13 & 0.878 \\
\hline Error & 16 & 206484 & 206484 & 12905 & & \\
\hline Total & 26 & 2034648 & & & & \\
\hline
\end{tabular}

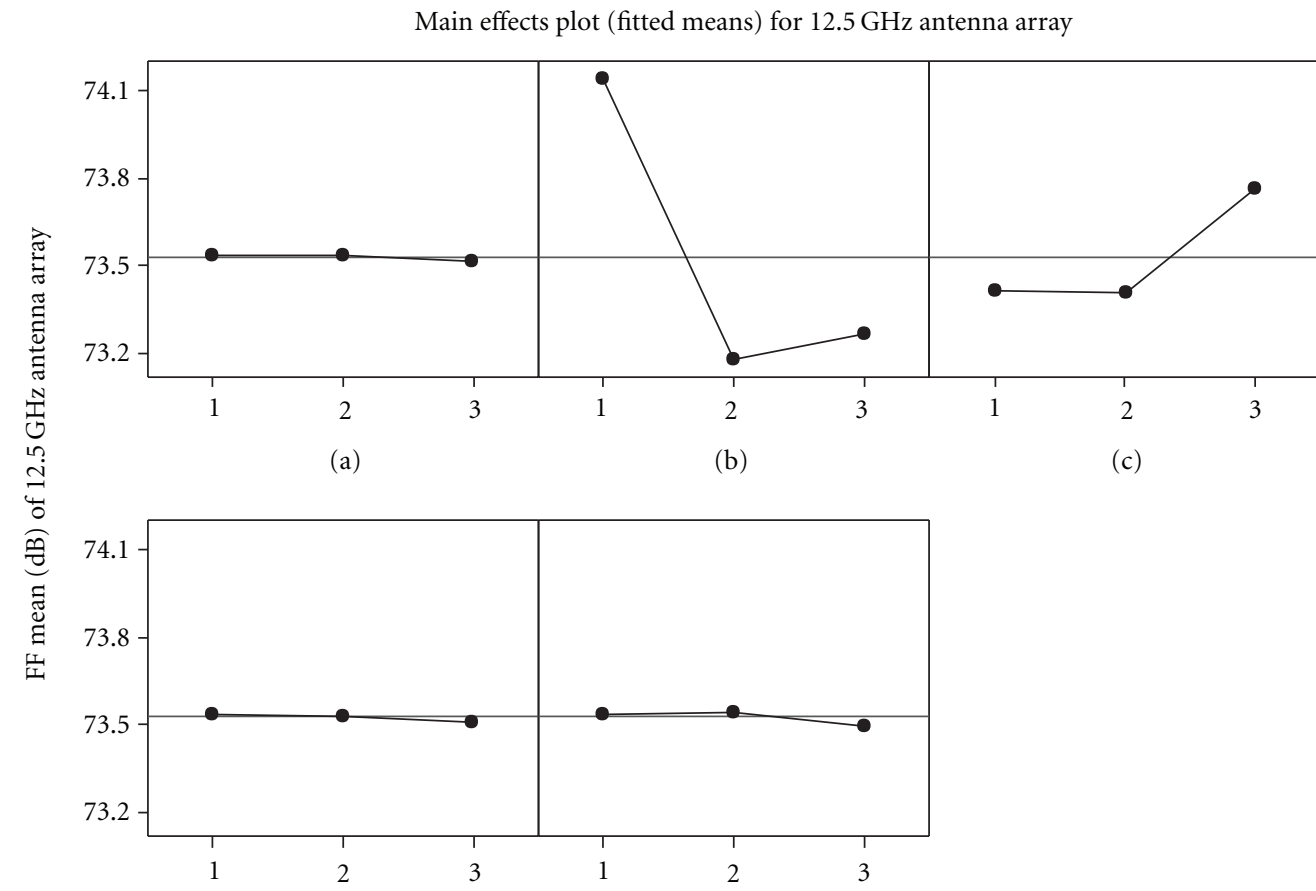

(d)

(e)

FIGURE 4: Main effects plot of the antenna's parameters variance at the achieved optimum level. 


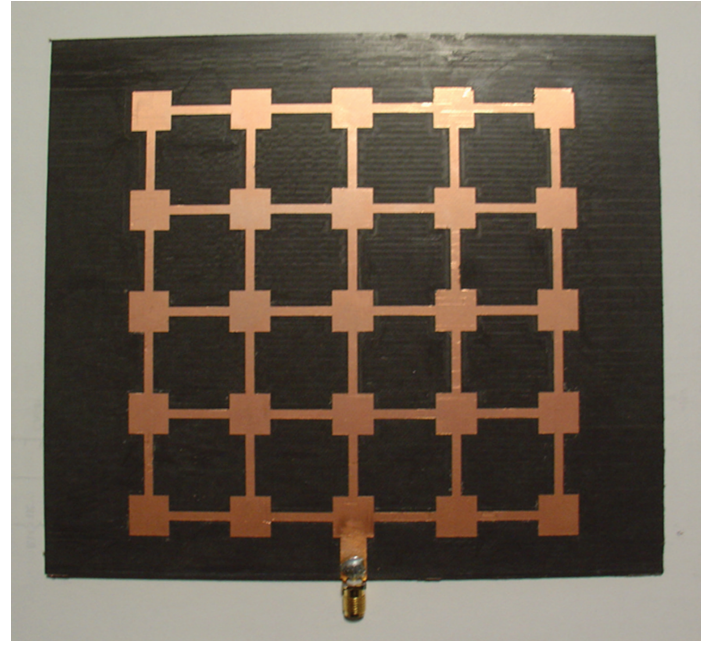

FIgURE 5: The fabricated array antenna.

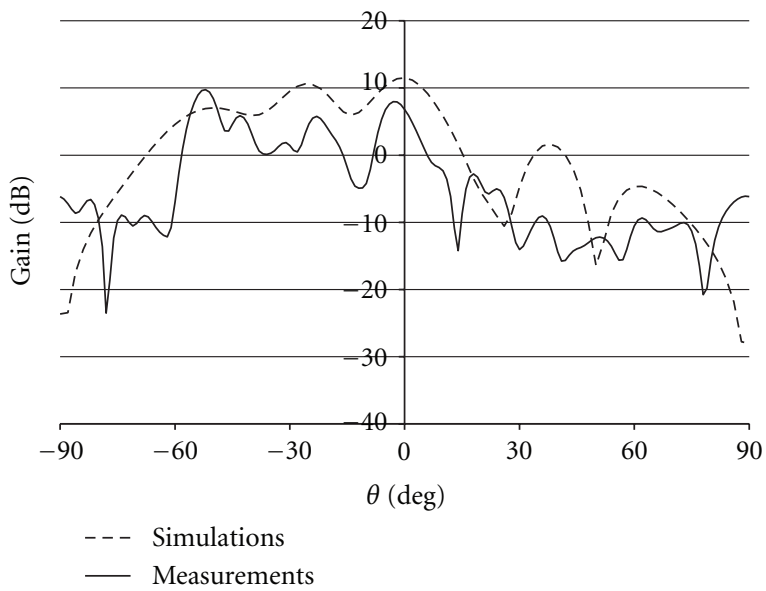

FIGURE 6: Radiation pattern on plane A $\left(\varphi=0^{\circ}, \theta j\left(-90^{\circ} \leq \theta j \leq\right.\right.$ $\left.90^{\circ}\right)$ ).

a statistical analysis of variance (ANOVA) using MINITAB 14 software package.

To execute this analysis, Table II, which includes the levels status of $\mathrm{OA}_{27}(35)$ corresponding to the antenna parameters, is transferred to a Minitab worksheet where the five parameters model has been assigned to the five columns of the parameters $(A, B, C, D, E)$. The results of the final iteration of the Taguchi's optimization procedure (20. $\log 10$ (FF) values) have been assigned to the next column as response and have been named as $12.5 \mathrm{GHz}$ antenna array.

According to the results which are illustrated in Table 4, only two of the parameters (B. connector length and C. connector width) are significant in the final iteration with a predefined significance level $\alpha>0.005$, and they are responsible for the $83.15 \%$ of the total variability of the mean value. Special care should be taken during the fabrication procedure of the antenna to keep these two parameters close to the optimum values in order to achieve a robust device.

The analysis of variance table gives, for each term in the model, the degrees of freedom (DF), the sequential sums of squares (Seq SSs), the adjusted (partial) sums of squares

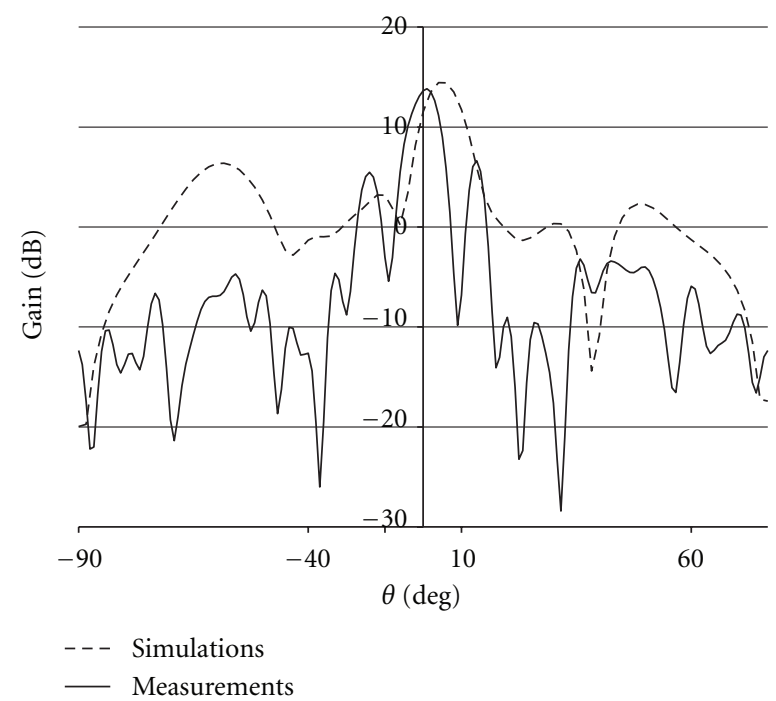

FIGURE 7: Radiation pattern on plane B $\left(\varphi=90^{\circ}, \theta j\left(-90^{\circ} \leq \theta j \leq\right.\right.$ $\left.90^{\circ}\right)$ ).

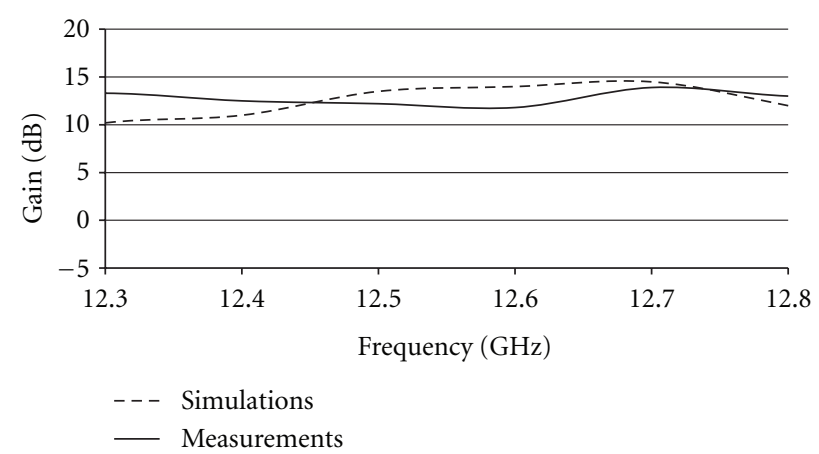

Figure 8: Comparison between measurements and simulations results for the antenna Gain at $\theta, \varphi=0^{\circ}$ for the frequency range 12.3-12.8 GHz.

(Adj SSs), the adjusted means squares (Adj MSs), and the $F$-statistic from the adjusted means squares and its $P$-value, which shows the significance of each parameter within its levels under a predefined level of significance. The same results from ANOVA are presented graphically with the main effects plot (fitted means $(\mathrm{dB})$ ) in Figure 4.

Taking into account the above precautions, the antenna array with its optimum dimensions has been fabricated on RT/duroid 5880 microwave laminate, as shown in Figure 5.

Simulations have been carried out using FEKO software package, and the results show high gain $\approx 15 \mathrm{~dB}$, as shown in Figures 6 and 7 for planes $\mathrm{A}$ and $\mathrm{B}$, high SLL $\approx 13 \mathrm{~dB}$ in B-plane, and very good matching $S 11 \approx-7 \mathrm{~dB}$ for the central frequency of $12.5 \mathrm{GHz}$. A small asymmetry has been observed in the pattern of plane B (Figure 7), due to asymmetric current distributions as shown in Figure 4. An array design with a feeding arrangement at the centre element will eliminate this problem. Also, a higher gain closer to $18-20 \mathrm{~dB}$ was not achieved as a nonuniform illumination resulted from this simple array configuration. 


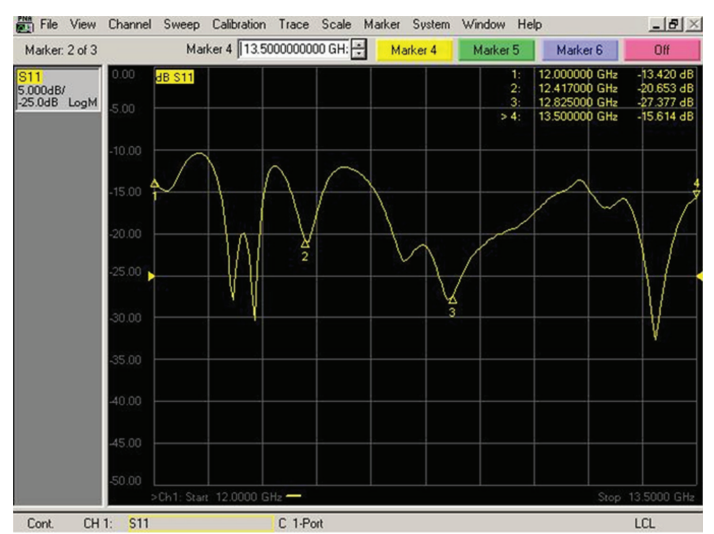

FIGURE 9: Network analyzer measurements as regards $S 11$.

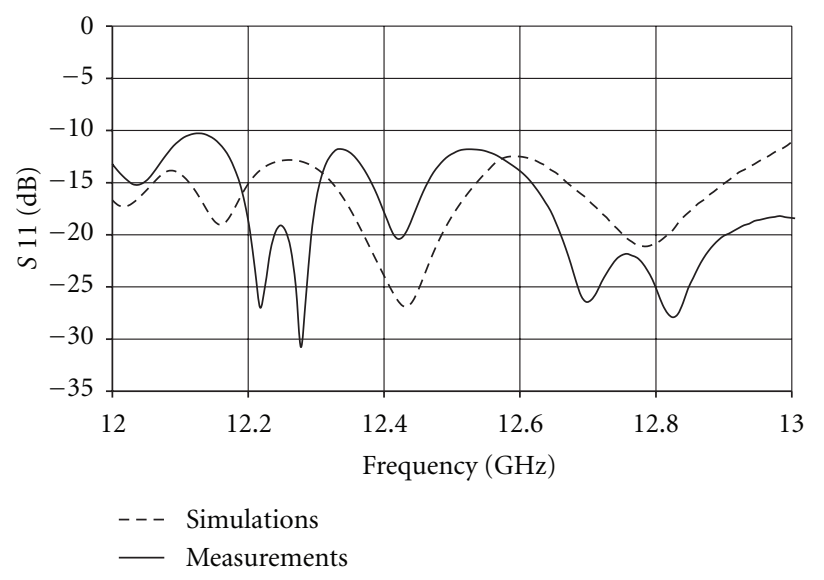

Figure 10: Comparison between measurements and simulations results for $S 11$.

The antenna array gain has been simulated as well as measured in the frequency range 12 to $13 \mathrm{GHz}$, presenting excellent results in the pattern cut $\varphi j(0 \leq \varphi j \leq 2 \pi), \theta j=$ $0^{\circ}($ Gain $>10 \mathrm{~dB})$, as illustrated in Figure 8.

The measurements of $S 11$ have been carried out using a network analyzer (Agilent Network Analyzer N5230A) and shown in Figure 9. A comparison between the simulations and measurements results of $S 11$ has been performed, which shows good agreement, as illustrated in Figure 10.

\section{Conclusions}

Analytical description of the Taguchi statistical optimization method for electromagnetic applications has been presented and implemented in the design and fabrication of a $12.5 \mathrm{GHz}$ single feed $5 \times 5$ planar microstrip antenna array. Following this method, complicated optimization goals such as matching and high directivity and low SLL have been accomplished and verified by the agreement between simulations (FEKO) and measurements (Agilent Network Analyzer N5230A). Besides, the antenna array gain has been measured in the frequency range 12 to $13 \mathrm{GHz}$ indicating high gain between 12.3 and $12.8 \mathrm{GHz}$, in good agreement with theory and simulation results. Finally, the efficiency measured was very satisfactory throughout the frequency range tested. It has to be mentioned that no feeding networks are used in this configuration. In order to create a robust construction, a statistical analysis of variance (MINITAB) has been performed and the most significant parameters of the antenna design have been discovered. This work is still ongoing, and an extended version of Taguchi's optimization method is under consideration with a wider consideration area for each parameter, and the results will be presented in the near future.

\section{Acknowledgment}

The authors would like to thank Roger Corporation for providing the dielectric substrates.

\section{References}

[1] N. Damavandi and S. Safavi-Naeini, "Antenna optimization using a hybrid evolutionary programming method," in Proceedings of the IEEE Antennas and Propagation Society International Symposium and USNC/URSI Meeting, pp. 65-68, Washington, DC, USA, July 2005.

[2] A. Hoorfar, "Evolutionary programming in electromagnetic optimization: a review," IEEE Transactions on Antennas and Propagation, vol. 55, no. 3 I, pp. 523-537, 2007.

[3] R. L. Haupt, "Thinned arrays using genetic algorithms," IEEE Transactions on Antennas and Propagation, vol. 42, no. 7, pp. 993-999, 1994.

[4] M. Donelli, S. Caorsi, F. de Natale, M. Pastorino, and A. Massa, "Linear antenna synthesis with a hybrid genetic algorithm," in Proceedings of the Electromagnetics Research, (PIER' 04), pp. 122, 2004.

[5] K. V. Deligkaris, Z. D. Zaharis, D. G. Kampitaki, S. K. Goudos, I. T. Rekanos, and M. N. Spasos, "Thinned planar array design using boolean PSO with velocity mutation," IEEE Transactions on Magnetics, vol. 45, no. 3, Article ID 4787361, pp. 14901493, 2009.

[6] Z. Zaharis, D. Kampitaki, A. Papastergiou, A. Hatzigaidas, P. Lazaridis, and M. Spasos, "Optimal design of a linear antenna array under the restriction of uniform excitation distribution using a particle swarm optimization based method," WSEAS Transactions on Communications, vol. 6, no. 1, pp. 52-59, 2007.

[7] N. Dipak, S. Shyam, S. Pattnaik et al., "Design of a wideband microstrip antenna and the use of artificial neural networks in parameter calculation," IEEE Antennas and Propagation Magazine, vol. 47, no. 3, pp. 60-65, 2005.

[8] S. Lebbar, Z. Guennoun, M. Drissi, and F. Riouch, "A compact and broadband microstrip antenna design using a geometrical-methodology-based artifical neural network," IEEE Antennas and Propagation Magazine, vol. 48, no. 2, pp. 146-154, 2006.

[9] D. I. Karatzidis, T. V. Yioultsis, and T. D. Tsiboukis, "Gradientbased adjoint-variable optimization of broadband microstrip antennas with mixed-order prism macroelements," $A E U$ International Journal of Electronics and Communications, vol. 62, no. 6, pp. 401-412, 2008.

[10] R. Haupt, "Comparison between genetic and gradient-based optimization algorithms for solving electromagnetics problems," IEEE Transactions on Magnetics, vol. 31, no. 3, pp. 19321935, 1995. 
[11] N. D. Lopez-Rivera and R. A. Rodriguez-Solís, "Input impedance and resonant frequency characterization for folded slot antennas through DOE techniques," in Proceedings of the IEEE International Antennas and Propagation Symposium and USNC/CNC/URSI North American Radio Science Meeting, pp. 545-548, June 2003.

[12] G. Taguchi and Y. Yokoyama, Taguchi Methods: Design of Experiments, vol. 4 of Quality Engineering, Amer Supplier Institute, 1993.

[13] G. Taguchi, Taguchi's Quality Engineering Handbook, WileyInterscience, New York, NY, USA, 2004.

[14] W. C. Weng and C. T. M. Choi, "Optimization comparison between taguchi's method and PSO by design of a CPW slot antenna," in Proceedings of the IEEE International Symposium on Antennas and Propagation and USNC/URSI National Radio Science Meeting, (APSURSI '09), June 2009.

[15] A. D. MacDonald, "A modified Taguchi method for the design of broadband spiral cavity absorbers," in Proceedings of the Antennas and Propagation Symposium Digest, pp. 1180-1183, May 1990.

[16] W. C. Weng, F. Yang, and A. Z. Elsherbeni, "Linear antenna array synthesis using Taguchi's method: a novel optimization technique in electromagnetics," IEEE Transactions on Antennas and Propagation, vol. 55, no. 3 I, pp. 723-730, 2007.

[17] G. Xu, C. X. Tang, F. B. Meng, Y. Liao, Z. B. Yang, and P. Xie, "Optimization method for two-layer patches wideband antenna based on Taguchi's algorithm," Chinese Journal of Radio Science, vol. 24, no. 6, pp. 1060-1064, 2009.

[18] C. M. De J. van Coevorden, A. R. Bretones, M. F. Pantoja, S. G. García, A. Monorchio, and R. G. Martín, "A new implementation of the hybrid taguchi GA: application to the design of a miniaturized Log-Periodic Thin-Wire antenna," Applied Computational Electromagnetics Society Journal, vol. 24, no. 1, pp. 21-31, 2009.

[19] FEKO Suite 5.4, V.4.0.10.4014, EM software \& systems-S.A. (Pty) Ltd, http://www.feko.info/.

[20] C. A. Balanis, Antenna Theory, Analysis and Design, John Wiley \& Sons, New York, NY, USA, 3rd edition, 2005.

[21] R. Garg, P. Bhartia, J. Bahl, and A. Ittipiboon, Microstrip Antenna Design Handbook, Artech House, London, UK, 2001. 

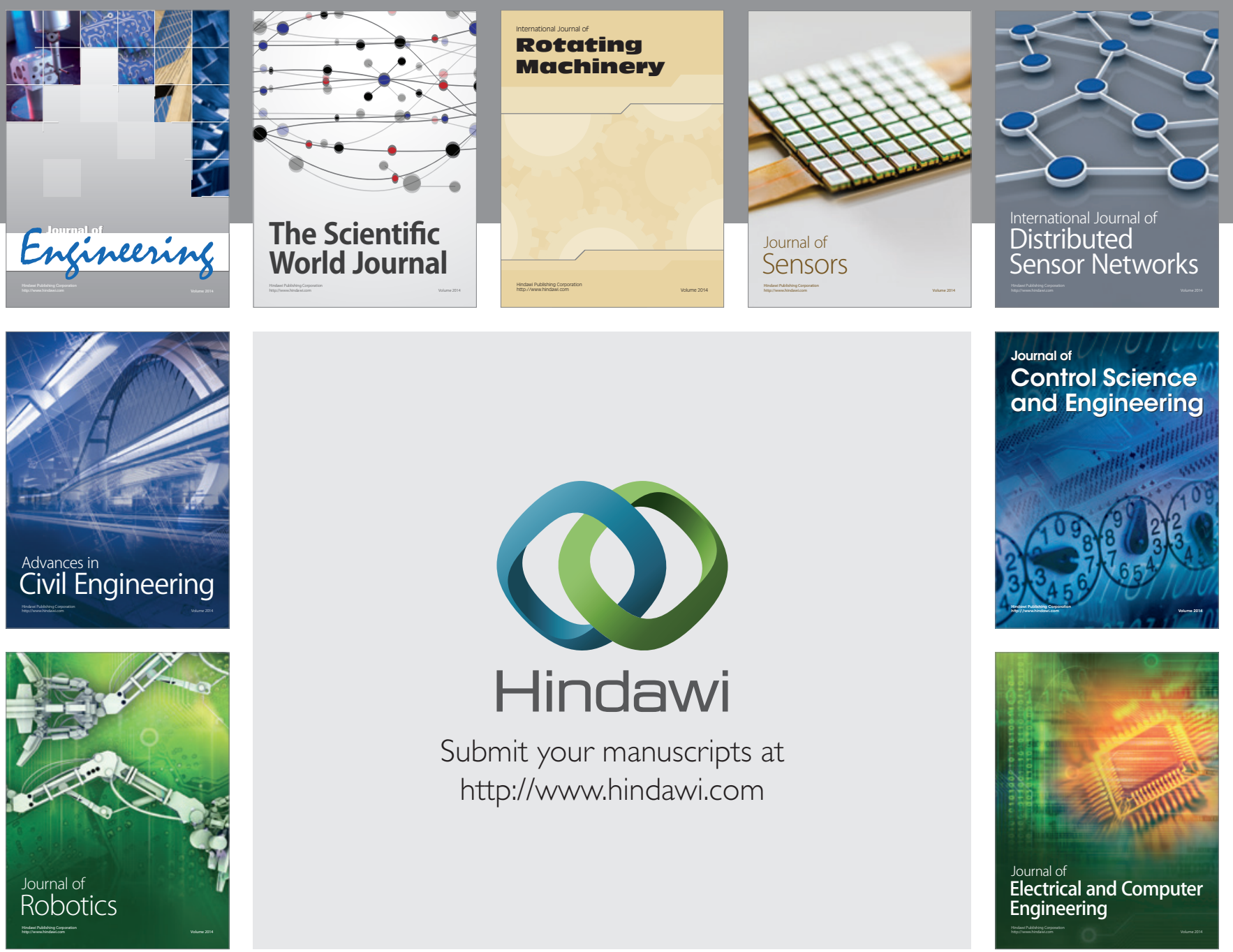

Submit your manuscripts at

http://www.hindawi.com
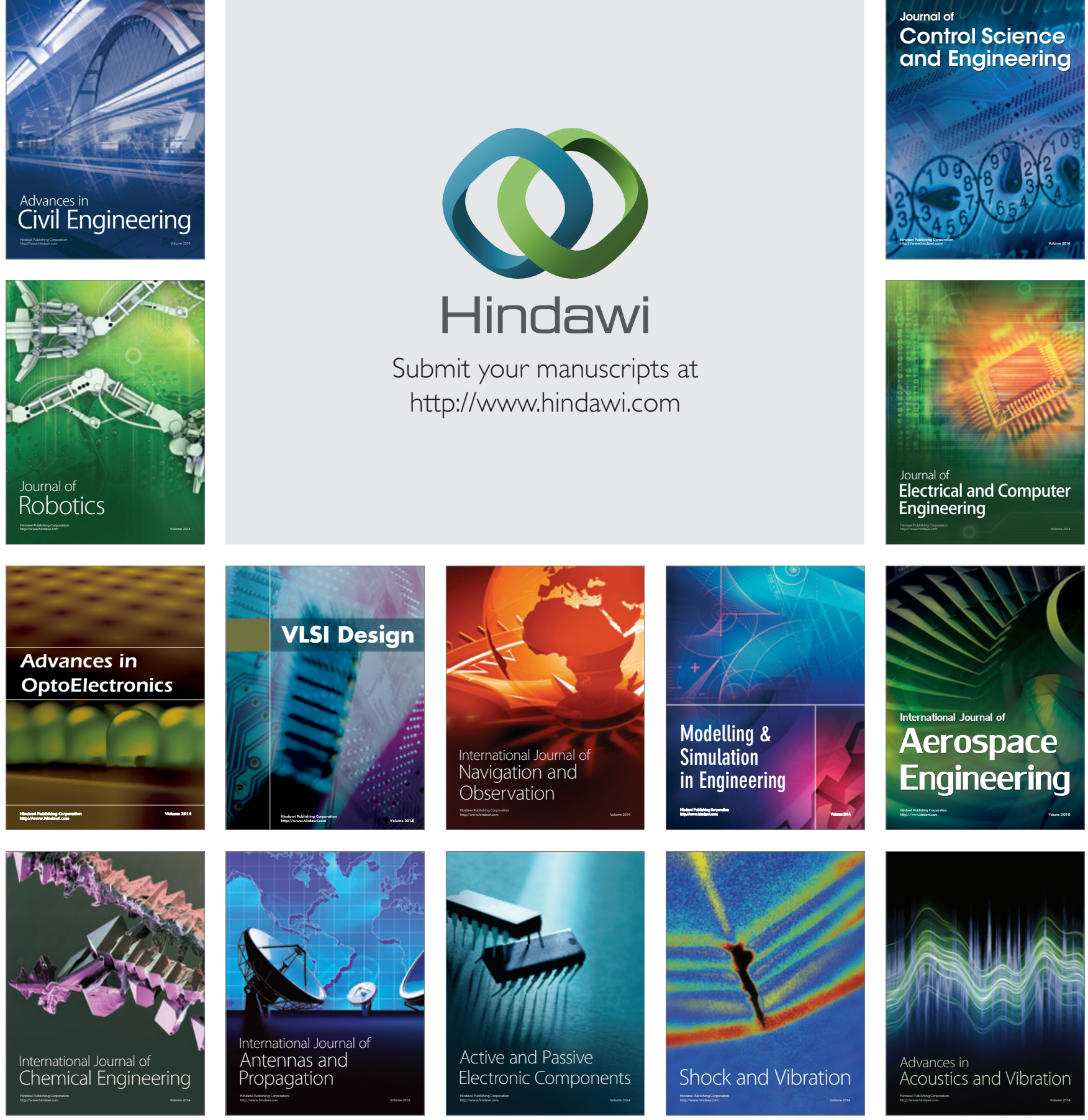